\title{
REVIEW
}

\section{Expectations of biologically active compounds of the genus Magnolia in biomedicine}

\author{
Jiří Patočka $^{1}$, Jiří Jakl $^{2}$, Anna Strunecká ${ }^{3}$ \\ ${ }^{1}$ Department of Radiology and Toxicology, Faculty of Health and Social Studies, University of South Bohemia, \\ České Budějovice, Czech Republic \\ ${ }^{2}$ Department of Dendrology and Forest Tree Breeding, Faculty of Forestry and Environment of the Czech \\ University of Agriculture Prague, Czech Republic \\ ${ }^{3}$ Department of Physiology and Developmental Biology, Faculty of Science, Charles University, Prague, Czech \\ Republic
}

Received $18^{\text {th }}$ July 2006.

Published online $20^{\text {th }}$ October 2006.

\begin{abstract}
Summary
Magnolia bark is a highly aromatic herbal material obtained from Magnolia officinalis (and other species) of the family Magnoliaceae. In traditional oriental herbal medicine, particularly Chinese medicine, this drug is used for many purposes, especially as a mild tranquillizer. The principal active compounds are the biphenol compounds, magnolol and honokiol, together with other biologically active compounds, which exert numerous and diverse pharmacological actions. Recent research has produced further evidence for the mechanism of their anti-inflammatory, anti-oxidant, antimicrobial, and antitumour activities, and these will be outlined in this review.
\end{abstract}

Keywords: herbal tranquillizer - honokiol - Magnolia - magnolol - obovatol - pharmacology

\section{INTRODUCTION}

The genus Magnolia is representative of the ancient family Magnoliaceae, which has been in existence since the Tertiary period and consists of 120-130 species. Magnolia bark is a traditional Chinese medicine, known under the name houpu (from Magnolia officinalis), that has been used for

Jiří Patočka, Department of Radiology and Toxicology, Faculty of Health and Social Studies, University of South Bohemia České Budějovice, 37001 České Budějovice, Czech Republic

ㅁ. prof.patocka@gmail.com

ㅇㅛㅛ +0420494661014 thousands of years to treat "stagnation of qi" (low energy), asthma, digestive problems, and emotional distress. Magnolia bark is used as a general antistress and anti-anxiety agent. Magnolia has also been traditionally used to treat breast cancer.

Hоири is an official herb in the Pharmacopoeia of the People's Republic of China (Pharmacopoeia 1988); the herb is sometimes called chuan houpu, because it originally came from the Sichuan area of China. Because traditional Chinese medicine is becoming increasingly popular in many medical contexts in other parts of the world, particularly among patients with cancer, it is important that recent research demonstrates the relevant pharmacological effects of various magnolias and the main pharmacologically active compounds (Ikeda et al. 2003, Yang et al. 2003). Based on the 
experimental evidence available so far, it seems likely that Magnolia might be helpful in modern medicine (Patočka et al. 2002).

\section{BIOLOGY OF MAGNOLIA GENUS}

Various magnolias are distinguished by their many interesting biological features. Current research raises basic questions as to the definition of the genus itself. The genus Magnolia consists of about $120-130$ species and in the Tertiary period Magnolias were common in Europe (Azuma et al. 2001, Kim et al. 2001, Hunt et al. 1998). The majority of Magnolia species grow in the temperate and tropical zones of southeastern Asia, while other species grow in the New World. Magnolia officinalis is not the only recognised source of magnolia bark; other closely related Asian species of the section Rytidospermum Spach are also used in traditional medicine, such as the Japanese Magnolia obovata 'Wakoboku' (Ito et al. 1982) or the Chinese Magnolia rostrata. However, according to the IUCN Red List these are vulnerable species due to over-harvesting of the bark and habitat destruction (IUCN 2004). Magnolia bark was a common ingredient in many formulas, for example 'Saiboku-to' (Maruyana et al. 1998), 'Xiao Zhengqi Tang', 'Maziren Wan', 'Ping Wei San' and 'Shenmi Tang' (Hong-Yen 1980). Some species of Magnolia contain magnolol and honokiol, and others lignanoids (Hegnauer 1990). The best-known magnolia, Magnolia grandiflora, is a popular plant utilised in Mexican traditional medicine (Bastidas Ramírez et. al. 1998). Another form of Magnolia used in medicine is derived from the flower buds ( $\mathrm{Hu} 2000)$. Currently, China produces about 200 tons of Magnolia bark per year (Jinping 2000).

\section{CHEMISTRY OF MAGNOLIA GENUS}

The principal substantial compounds present in plants of the Magnoliaceae family are different phenolic compounds and terpenoids. Many phenolic compounds have been found in the leaves and bark; for example gallic acid, sennosides A and $\mathrm{B}$, hesperidin, naringin, syringin, and especially two neolignan compounds, magnolol (I) and honokiol (II) (Fig.1). The magnolol content of magnolia bark is generally in the range of $2-10 \%$, while honokiol tends to occur naturally at 1-5 percent in the dried bark. Magnolol and honokiol are without question pharmacologically the most meaningful constituents of magnolia bark (Watanabe et al. 1983, Liu et al. 2006). From the leaves and bark of $M$. obovata, the novel biphenyl ether lignans, obovatol (III) (Fig. 1) and obovatal were isolated (Ito et al. 1982), together with some sesquiterpene-neolignans, eudesobovatols A and B, eudesmagnolol, eudeshonokiols $\mathrm{A}$ and $\mathrm{B}$, clovanemagnolol, and caryolanemagnolol (Fukuyama et al. 1992).

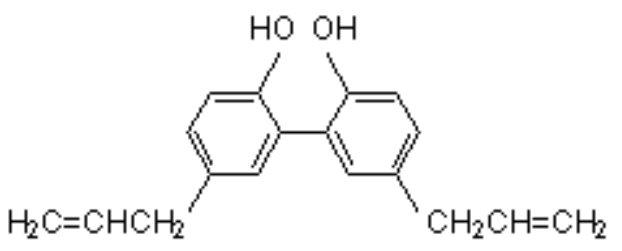

I

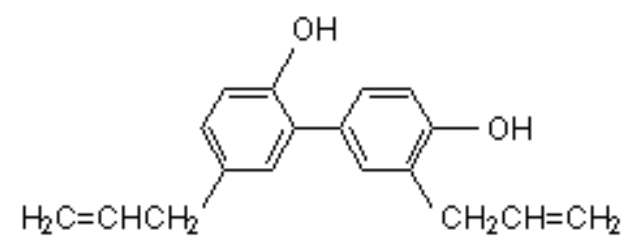

II

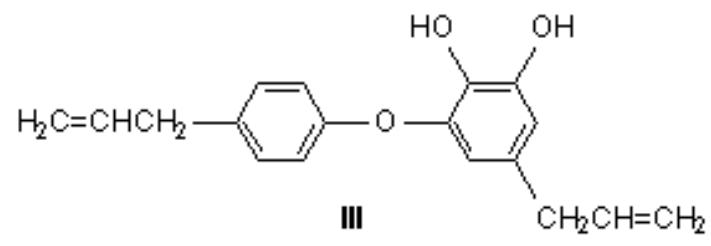

Fig. 1. Chemical structures of three principal magnolia lignans: magnolol (I), honokiol (II), and obovatol (III)

Several monoterpenes and sesquiterpenoids have been obtained from the leaves of Magnolia grandiflora L, and, on the basis of spectral evidence, their structures determined as $\alpha$ - and $\beta$ pinenes, $\beta$-eudesmol and bornyl acetate

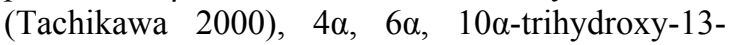
acetoxyguaia-11-ene and 12,13-diacetoxyguaia- $4 \alpha$, $6 \alpha, 10 \alpha, 11$-tetraol (Yang et al. 1994). In addition, the known sesquiterpenoid magnograndiolide was also obtained (Luo et al. 2001). Recently, a new sesquiterpenoid was obtained from the leaves of Magnolia delavayi. Its structure was determined as $8 \beta$-acetoxy-10 $\alpha$-ethyloxy-guaia- $4 \alpha$, 11-diol (Cao et al. 2004). A new tricyclo [4.2.0.0(2,8)] octanetype neolignan, 6-allyl-7- (3,4-dimethoxyphenyl)2,3-dimethoxy-8-methyl-tricyclo [4.2.0.0(2,8)] oct3 -en-5-one, together with 15 known lignan and neolignan derivatives have been isolated from the flower buds of Magnolia denudata DESR (Li et al. 2005). 


\section{PHARMACOLOGY OF MAGNOLIA GENUS}

\section{Pharmacology of magnolol and honokiol}

Magnolol and honokiol, two major phenolic constituents of Magnolia species which are abundantly found in the medicinal plants $M$. officinalis and $M$. obovata, show multiple pharmacological effects (Chen et al. 2006). Research has elucidated the underlying mechanism of some of their anti-inflammatory and antioxidative effects. It has been found, for example, that magnolol is 1000 -fold more potent than $\alpha$ tocopherol in inhibiting lipid peroxidation in rat mitochondria (Chang et al. 2003). All active Magnolia constituents (magnolol, honokiol, obovatol) showed weak inhibition for inducible NO synthase (iNOS) activity, but potent inhibition of iNOS induction and activation of nuclear factorkappa B (Matsuda et al. 2001). They also inhibit rat liver acyl-CoA: cholesterol acyltransferase (ACAT) with $\mathrm{IC}_{50}$ values of 42,71 , and $86 \mu \mathrm{M}$, respectively (Kwon et al. 1997). Honokiol may protect the myocardium against ischemic injury and suppress ventricular arrhythmia during ischemia (Tsai et al. 1999). The mechanism of anxiolytic activity of various Magnolia extracts has been studied. The observed antimicrobial activity demonstrates the potential of Magnolias to be an adjunct in the treatment of periodontitis (Ho et al. 2001).

\section{Anti-inflammatory activity}

The reactive oxygen species produced by neutrophils contribute to the pathogenesis of focal cerebral ischemia/reperfusion injury and signal the inflammatory response. Recently it was shown that honokiol has a protective effect against focal cerebral ischemia/reperfusion injury in rats that paralleled a reduction in reactive oxygen species production by neutrophils (Liou et al. 2003). To elucidate the underlying mechanism(s) of the antioxidative effect of honokiol, peripheral neutrophils isolated from rats were activated with phorbol-12-myristate-13-acetate (PMA) or Nformyl-methionyl-leucyl-phenylalanine (fMLP) in the presence or absence of honokiol. Liou et al. (2003) suggested that honokiol inhibited PMA- or fMLP-induced reactive oxygen species production by neutrophils by three distinct mechanisms: (i) honokiol diminished the activity of assembledNADPH oxidase, a major reactive oxygen species producing enzyme in neutrophils by $40 \%$ without interfering with its protein kinase $\mathrm{C}$ (PKC)dependent assembly; (ii) honokiol inhibited two other important enzymes for reactive oxygen species generation in neutrophils, i.e., myeloperoxidase and cyclooxygenase, by $20 \%$ and $70 \%$, respectively; (iii) honokiol enhanced by $30 \%$, the activity of glutathione (GSH) peroxidase, an enzyme that triggers the metabolism of hydrogen peroxide $\left(\mathrm{H}_{2} \mathrm{O}_{2}\right)$. These data suggested that honokiol, acting as a potent reactive oxygen species inhibitor/scavenger, could achieve its focal cerebral ischemia/reperfusion injury protective effect by modulating enzyme systems related to reactive oxygen species production or metabolism, including NADPH oxidase, myeloperoxidase, cyclooxygenase, and GSH peroxidase in neutrophils.

Magnolol is hypothesized to suppress TNFalpha production after the endotoxin tolerance induced by sublethal hemorrhage (SLH) and to alter or attenuate subsequent endotoxin tolerance (Liou et al. 2003). Recent results show that the anti-inflammatory effects of magnolol and honokiol are mediated through inhibition of the downstream pathway of MEKK-1 in NF-kappaB activation signalling (Lee et al. 2005). Plasma and tissue TNF-alpha increased after sublethal hemorrhage (SLH); this increase was significantly suppressed by magnolol. Lipid peroxidation and SOD activity increased after SLH; magnolol suppressed the lipid peroxidation but not the SOD activity. In conclusion, magnolol induces an antiinflammatory response and provides early protection against endotoxin challenge following $\mathrm{SLH}$; however, magnolol attenuates the protraction of endotoxin tolerance and inhibits late protection against endotoxin challenge following SLH (Shih et al. 2004). The anti-inflammatory and neuroprotective effects of magnolol have been demonstrated by other authors (Wang et al. 1995; Lee et al. 2000, Park et al. 2004, Matsui et al. 2005, Lin et al. 2006).

Magnolol inhibited mouse hind-paw oedema induced by carrageenan, and polymyxin $\mathrm{B}$, and reversed the passive Arthus reaction. The recovered myeloperoxidase activity in the oedematous paw was significantly decreased in mice pretreated with magnolol. Suppression of oedema was demonstrated not only in normal mice but also in adrenalectomized animals. Magnolol was less potent in reducing PGD2 formation in rat mast cells than indomethacin. Unlike dexamethasone, magnolol did not increase the liver glycogen level. The results suggest that the anti-inflammatory effect of magnolol was neither mediated by glucocorticoid activity, nor through releasing steroid hormones from the adrenal gland. It is proposed that the action of magnolol is dependent on reducing the level of eicosanoid mediators (Wang et al. 1992).

\section{Antioxidant activity}

Magnolol induces apoptosis in rat vascular smooth muscle cells (VSMCs) via the mitochondrial death pathway. This effect is mediated through downregulation of Bcl-2 protein levels, both in vivo and in vitro. Magnolol thus shows potential as a novel therapeutic agent for the treatment of atherosclerosis and re-stenosis (Chen et al. 2003). 
Magnolol suppressed thromboxane $\mathrm{B}_{2}\left(\mathrm{TXB}_{2}\right)$ and leukotriene $\mathrm{B}_{4}\left(\mathrm{LTB}_{4}\right)$ formation in A23187stimulated rat neutrophils. Maximum inhibition was obtained with about $10 \mu \mathrm{M}$ magnolol. Magnolol was more effective in the inhibition of cyclooxygenase (COX) activity than in the inhibition of 5-lipoxygenase (5-LO) activity, as assessed by means of enzyme activity determination in vitro and $\mathrm{COX}$ and 5-LO metabolic capacity analyses in vivo. Magnolol alone stimulated cytosolic phospholipase $\mathrm{A}_{2}$ $\left(\mathrm{cPLA}_{2}\right)$ phosphorylation and the translocation of 5-LO and $\mathrm{cPLA}_{2}$ to the membrane, and evoked arachidonic acid (AA) release. These results indicate that magnolol inhibits the formation of prostaglandins and leukotrienes in A23187stimulated rat neutrophils, probably through a direct blockade of COX and 5-LO activities (Hsu et al. 2004). The hepatoprotective effects of honokiol and magnolol on oxidative stress induced by tertbutylhydroperoxide were probably the result of their antioxidant activity. Honokiol and magnolol also had a protective effect against Dgalactosamine-induced hepatotoxicity, which was used as an alternate model to oxidative stress, acting by inhibiting intracellular GSH depletion (Park et al. 2003). Recently a novel synthetically prepared magnolol derivative, 3,3'-bis-allylmagnolol, was developed as a potential antioxidant for certain diseases ( $\mathrm{Li}$ et al. 2003).

\section{Anxiolytic activity}

The bark of the root and stem of various Magnolia species has been used in Traditional Chinese Medicine to treat a variety of disorders including anxiety and nervous disturbances. Honokiol and magnolol have been identified as modulators of the GABA(A) receptors in vitro (Squires et al. 1999, Ai et al. 2001). The possible selectivity of honokiol and magnolol on GABA(A) receptor subtypes was demonstrated in a study using ${ }^{3} \mathrm{H}$-muscimol and ${ }^{3} \mathrm{H}$-flunitrazepam binding assays on various rat brain membrane preparations and human recombinant GABA(A) receptor subunit combinations. These results indicate that honokiol and magnolol have some selectivity on different GABA(A) receptor subtypes, which could be responsible for the reported in vivo effects of these two compounds.

The anxiolytic effect of honokiol, evaluated by means of an elevated plus-maze test, was at least 5000 times more potent than the compound preparation 'Saiboku-to' when mice were treated orally for seven days, and was comparable with the effect of benzodiazepines (Maruyama et al. 1998). Kuribara et al. (1999) compared the anxiolytic potentials of honokiol and water extracts of three Magnolia samples using an improved elevated plus-maze in mice. Their results suggest that honokiol is the major constituent responsible for the observed anxiolytic effect of the water extract of Magnolia, and that the other components, including magnolol, scarcely influence the effect of honokiol.

\section{Antiarrhythmic activity}

Tsai et al. (1996) demonstrated that honokiol may protect the myocardium against ischemic injury and suppress ventricular arrhythmia during ischemia and reperfusion. The experimental ventricular arrhythmia induced by coronary ligation of rats for 30 min were significantly reduced after intravenous pre-treatment (15 $\mathrm{min}$ before coronary ligation) with $10^{-7} \mathrm{~g} / \mathrm{kg}$ magnolol or $10^{-7} \mathrm{~g} / \mathrm{kg}$ honokiol. However, the antiarrhythmic effect of magnolol or honokiol could be abolished with the pre-treatment of $1 \mathrm{mg} / \mathrm{kg}$ nitric oxide inhibitor (L-NAME), but not with pre-treatment of $100 \mathrm{mg} / \mathrm{kg}$ aspirin. The abolishment of the beneficial effects of magnolol and honokiol on the myocardium by L-NAME, rather than aspirin, suggests the involvement of an increased nitric oxide synthesis in the protection offered by magnolol and honokiol against arrhythmia during myocardial ischemia (Tsai et al. 1999).

\section{Antimicrobial activity}

Three phenolic constituents of Magnolia grandiflora L. were shown to possess significant antimicrobial activity using an agar well diffusion assay. Magnolol, honokiol, and 3.5'-diallyl-2'hydroxy-4-methoxybiphenyl exhibited significant activity against Gram-positive and acid-fast bacteria and fungi (Clark et al. 1981). Magnolol and honokiol have an antimicrobial activity against numerous microorganisms such as Porphyromonas gingivalis, Prevotella intermedia, Actinobacillus actinomycetemcomitans, Capnocytophaga gingivalis, Veillonella disper, Micrococcus luteus, and Bacillus subtilis (Chang et al. 1998, Ho et al. 2001).

Both biphenolic compounds, although less potent than chlorhexidine, show a significant antimicrobial activity against these microorganisms, and a relatively low cytotoxic effect on human gingival cells. Thus, it is suggested that magnolol and honokiol might have a potential therapeutic use as a safe oral antiseptic for the prevention and the treatment of periodontal disease (Chang et al. 1998, Ho et al. 2001). Magnolol from Magnolia officinalis (cortex) potently inhibited the growth of Helicobacter pylori (Bae et al. 1998).

\section{Antitumor activity}

The neolignans magnolol and honokiol have been reported to inhibit the growth of several tumour cell lines, both in vitro and in vivo (Kong et al. 2005). Magnolol has been reported to have anticancer activity (Lin et al. 2001). Magnolol at very low concentrations inhibited DNA synthesis and 
decreased cell number in cultured human cancer cells (COLO-205 and Hep-G2) in a dose-dependent manner, but not in human untransformed cells such as keratinocytes, fibroblasts, and human umbilical vein endothelial cells (HUVEC). Magnolol was not cytotoxic at these concentrations and this indicates that it may have an inhibitory effect on cell proliferation in the subculture cancer cell lines (Lin et al. 2002). Magnolol possesses the ability to inhibit tumour growth due to the induction of apoptosis with the activation of caspases (Ikeda and Nagase 2002) and a strong antimetastatic effect due to its ability to inhibit tumour cell invasion (Ikeda et al. 2003). Magnolol induced the reduction of mitochondrial transmembrane potential and the release of cytochrome $\mathrm{C}$ into the cytoplasm. Magnolol-induced apoptotic signalling appears to be carried out through mitochondrial alternations to caspase-9, and then downstream effector caspases are activated sequentially. Magnolol could be thus a potentially effective drug for the adjunctive treatment of leukaemia, with low toxicity to normal blood cells (Zhong et al. 2003). These findings warrant further investigation.

Recently Fong et al., (2005) discovered that magnolol and honokiol enhance HL-60 cell differentiation initiated by low doses of 1.25 dihydroxyvitamin D3 (VD3) and all-trans-retinoic acid (ATRA). Cells expressing membrane differentiation markers CD11b and CD14 were increased from $4 \%$ in the non-treated control to 8 $16 \%$ after being treated with $10-30 \mu \mathrm{M}$ magnolol or honokiol. It is evident that both these neolignans are potential differentiation enhancing agents, which may allow the use of low doses of VD3 and ATRA in the treatment of acute promyelocytic leukaemia (Fong et al. 2005). Honokiol demonstrated weak activity against HIV-1 in human lymphocytes (Amblard et al. 2006).

Magnolol is a strong 11-beta-hydroxysteroid dehydrogenase (11-beta-HSD) inhibitor and, like glycyrrhetinic acid, another 11beta-HSD inhibitor isolated from licorice, induces apoptosis of murine thymocytes via the accumulation of corticosterone. Magnolol has inhibited the enzyme activity in the kidney $(\mathrm{P}<0.0001)$ and thymus $(\mathrm{P}<0.002)$, while the activity in the liver was not affected. Blood concentrations of corticosterone in the magnololtreated mice were unexpectedly lower than those in the control animals $(\mathrm{P}<0.002)$. This means that the inhibition of 11 beta-HSD by magnolol did not increase the systemic level of corticosterone which is relevant to thymocyte apoptosis (Horigome et al. 2001).

\section{Pharmacology of obovatol}

The biphenyl ether lignan obovatol from M. obovata (Ito et al. 1982) is slightly different from magnolol and honokiol not only chemically but also pharmacologically. Obovatol inhibited the chitin synthase 2 activity of Saccharomyces cerevisiae with an $\mathrm{IC}_{50}$ of $38 \mu \mathrm{M}$. Its derivative, tetrahydroobovatol, inhibited chitin synthase 2 activities under the same conditions with an $\mathrm{IC}_{50}$ of $59 \mu \mathrm{M}$. These compounds exhibited no inhibitory activity for chitin synthase 3, and showed less inhibitory activity for chitin synthase 1 than for chitin synthase $2\left(\mathrm{IC}_{50}>1 \mathrm{mM}\right)$. These results indicated that obovatol and tetrahydroobovatol are specific inhibitors of chitin synthase 2 . Furthermore, obovatol and tetrahydroobovatol showed antifungal activities against various pathogenic fungi, with a particularly strong inhibitory activity against Cryptococcus neoformans (MIC $7.8 \mathrm{mg} / \mathrm{L}$ ). The results indicate that obovatol and tetrahydroobovatol can potentially serve as antifungal agents (Hwang et al. 2002).

\section{TOXICOLOGY OF MAGNOLIA GENUS}

Magnolia extracts have a two thousand-year-old safety record for use as a Chinese medicine, (Bateman et al. 1998), and no significant toxicity or adverse effects have been reported so far, although no special chronic toxicological studies with magnolol, honokiol, and obovatol have been performed. Very small doses of magnolol and honokiol appear to be safe and effective for anxiety and depression. However, large doses may cause a sedative effect and interact with alcohol, increasing its effects, so driving or operating dangerous equipment should be avoided when taking larger doses of Magnolia extract. Further work on the toxicology and potential drug interactions of the constituents of Magnolia need to be performed, in order that the useful properties of Magnolia species can be realised.

\section{ACKNOWLEDGEMENT}

Preparation of manuscript was supported by A. Alzheimer Award of Academia Medica Pragensis, 2004.

\section{REFERENCES}

Ai J., Wang X., Nielsen M.: Honokiol and magnolol selectively interact with GABA(A) receptor subtypes in vitro. Pharmacology 63: 34-41, 2001.

Amblard F., Delinsky D., Arbiser J.L., Schinazi R.F.: Facile purification of honokiol and its 
antiviral and cytotoxic properties. J. Med. Chem. 49:3426-3427, 2006.

Azuma H., Garcia-Franco J.G., Rico-Gray V., Thien L.B.: Molecular phylogeny of the Magnoliaceae: the biogeography of tropical and temperate disjunctions. Am. J. Bot. 88: 2275-2285, 2001.

Bae E.A., Han M.J., Kim N.J., Kim D.H.: AntiHelicobacter pylori activity of herbal medicines. Biol. Pharm. Bull. 21:990-992, 1998.

Bastidas Ramírez B.E., Navarro Ruíz N., Quezada Arellano J.D. et al.: Anticonvulsant effects of Magnolia grandiflora L. in the rat. J. Ethnopharmacol. 61:143-152, 1998.

Bateman J., Chapman R.D., Simpson D.: Possible toxicity of herbal remedies. Scott. Med. J. 43:7-15, 1998.

Cao J.X., Lai G.F., Wang Y.F. et al.: A new sesquiterpenoid from Magnolia delavayi. Chin. Chem. Lett. 15:791-793, 2004.

Chang B., Lee Y., Ku Y. et al.: Antimicrobial activity of magnolol and honokiol against periodontopathic microorganisms. Planta Med. 64:367-369, 1998.

Chang C.P., Hsu Y.C., Lin M.T.: Magnolol protects against cerebral ischemic injury of rat heat stroke. Clin. Exp. Pharmacol. Physiol. 30: 387-392, 2003.

Chen C., Xu X., Zhu Y. et al.: Determination of honokiol and magnolol in cortex Magnoliae Officinalis by capillary electrophoresis with electrochemical detection. J. Pharm. Biomed. Anal. 41:1479-1484, 2006.

Chen J.H, Wu C.C., Hsiao G., Yen M.H.: Magnolol induces apoptosis in vascular smooth muscle. Naunyn Schmiedebergs Arch. Pharmacol. 368: 127-133, 2003.

Clark A.M., El-Feraly F.S., Li W.S.: Antimicrobial activity of phenolic constituents of Magnolia grandiflora L. J. Pharm. Sci. 70:951-952, 1981.

Fong W. F., Tse A. K., Poon K.H., Wang C.: Magnolol and honokiol enhance HL-60 human leukemia cell differentiation induced by 1,25 dihydroxyvitamin D3 and retinoic acid. Int. J. Biochem. Cell. Biol. 37:427-441, 2005.

Fukuyama Y., Otoshi Y., Miyoshi K. et al.: Neurotrophic sesquiterpene-neolignans from Magnolia obovata - structure and neurotrophic activity. Tetrahedron 48:377-392, 1992.

Hegnauer R.: Chemotaxonomie der Pflanzen 9; Birkhäuser Verlag, Basel, Boston, Berlin 1990.

Ho K.Y., Tsai C.C., Chen C.P. et al.: Antimicrobial activity of honokiol and magnolol isolated from Magnolia officinalis. Phytother. Res. 15: 139-141, 2001.

Hong-Yen H., Chau-Shin H.: Commonly Used Chinese Herb Formulas with Illustrations.
Oriental Healing Arts Institute, Long Beach, CA 1980.

Horigome H., Homma M., Hiran T. et al.: Magnolol from Magnolia officinalis inhibits 11 beta-hydroxysteroid dehydrogenase without increases of corticosterone and thymocyte apoptosis in mice. Planta Med. 67:33-37, 2001.

Hsu M.F., Lu M.C., Tsao L.T. et al.: Mechanisms of the influence of magnolol on eicosanoid metabolism in neutrophils. Biochem. Pharmacol. 67:831-840, 2004.

Hu S.: The Economic botany of Magnoliaceae. In: Liu Y., Fan H., Chen Z., et al. (eds). Proceedings of the Internetional Symposium on the Family Magnoliaceae, Science Press, Beijing 2000.

Hunt D.: Magnolias and their allies. The International Dendrology Society and the Magnolia Society, Milborne Port 1998.

Hwang E.I., Kwon B.M., Lee S.H. et al.: Obovatols, new chitin synthase 2 inhibitors of Saccharomyces cerevisiae from Magnolia obovata. J. Antimicrob. Chemother. 49:95101, 2002.

Ikeda K., Nagase H.: Magnolol has the ability to induce apoptosis in tumor cells. Biol. Pharm. Bull. 25:1546-1549, 2002.

Ikeda K., Sakai Y., Nagase H.: Inhibitory effect of magnolol on tumour metastasis in mice. Phytother. Res. 17:933-937, 2003.

Ito K., Iida T., Ichino K. et al. Obovatol and obovatal, novel biphenyl ether lignans from the leaves of Magnolia obovata Thunb. Chem. Pharm. Bull. 30:3347-3353, 1982.

IUCN Red List of Threatened Species. IUCN 2004. http://www.iucnredlist.org/

Jinping S.: Jingning Magnolia officinalis development project, Jingning Science and Technology Development Department, Zhejiang, China 2000.

Kim S., Mark W.C., Parks C.R.: Phylogentic relationships in family Magnoliaceae inferred from ndhF sequences. Am. J. Bot. 88:717-728, 2001.

Kong Z.L., Tzeng S.C., Liu Y.C.: Cytotoxic neolignans: an SAR study. Bioorg. Med. Chem. Lett. 15:163-166, 2005.

Kwon B.M., Kim M.K., Lee S.H. et al.: Acyl-CoA cholesterol acyltransferase inhibitors from Magnolia obovata. Planta Med. 63: 550-551, 1997.

Kuribara H., Kishi E., Hattori N. et al.: Application of the elevated plus-maze test in mice for evaluation of the content of honokiol in water extracts of magnolia. Phytother. Res. 13:593596, 1999.

Lee J., Jung E., Park J. et al.: Anti-inflammatory effect of magnolol and honokiol are mediated through inhibition of the downstream pathway 
of MEKK-1 in NF-kappa B activation signaling. Planta Med. 71:338-343, 2005.

Lee M.M., Huang H.M., Hsieh M.T. et al.: Antiinflammatory and neuroprotective effects of magnolol in chemical hypoxia in rat cultured cortical cells in hypoglycemic media. Chin. J. Physiol. 43:61-67, 2000.

Li C.Y., Wang Y., Hu M.K.: Allylmagnolol, a novel magnolol derivative as potent antioxidant. Bioorg. Med. Chem. 11:36653671, 2003.

Li J., Tanaka M., Kurasawa K. et al.: Lignan and neolignan derivatives from Magnolia denudata. Chem. Pharm. Bull. (Tokyo) 53: 235-237, 2005.

Lin S.Y., Chang Y.T., Liu J.D. et al.: Molecular mechanisms of apoptosis induced by magnolol in colon and liver cancer cells. Mol. Carcinog. 32:7-83, 2001.

Lin S.Y., Liu J.D., Chang H.C. et al.: Magnolol suppresses proliferation of cultured human colon and liver cancer cells by inhibiting DNA synthesis and activating apoptosis. J. Cell. Biochem. 84:532-544, 2002.

Lin Y.R., Chen H.H., Ko C.H., Chan M.H.: Neuroprotective activity of honokiol and magnolol in cerebellar granule cell damage. Eur. J. Pharmacol. 537: 64-69, 2006.

Liou K.T., Shen Y.C., Chen C.F. et al.: The antiinflammatory effect of honokiol on neutrophils: mechanisms in the inhibition of reactive oxygen species production. Eur. J. Pharmacol. 475:19-27, 2003.

Liu L., Wu X., Fan X., Hu Z.: Separation and determination of honokiol and magnolol in herbal medicines by flow injection-capillary electrophoresis. Anal. Bioanal. Chem. 384: 1533-1539, 2006.

Luo X.D., Wu S.H., Ma Y.B. et al.: Sesquiterpenoids from Magnolia grandiflora. Planta Med. 67:354-357, 2001.

Maruyama Y., Kuribara H., Morita M. et al.: Identification of magnolol and honokiol as anxiolytic agents in extracts of Saiboku-to, an oriental herbal medicine. J. Nat. Prod. 61:135138, 1998.

Matsuda H., Kageura T., Oda M. et al.: Effects of constituents from the bark of Magnolia obovata on nitric oxide production in lipopolysaccharide-activated macrophages. Chem. Pharm. Bull. (Tokyo) 49:716-720, 2001.

Matsui N., Nakashima H., Ushio Y. et al.: Neurotrophic effect of magnolol in the hippocampal CA1 region ofsenescenceaccelerated mice (SAMP1). Biol. Pharm. Bull. 28:1762-1765, 2005.

Park E.J., Zhao Y.Z., Na M. et al.: Protective effects of honokiol and magnolol on tertiary butyl hydroperoxide- or D-galactosamine- induced toxicity in rat primary hepatocytes. Planta Med. 69:33-37, 2003.

Park J., Lee J., Jung E. et al.: In vitro antibacterial and anti-inflammatory effects of honokiol and magnolol against Propionibacterium sp. Eur. J. Pharmacol. 496:189-195, 2004.

Patočka J., Strunecká A., Jakl J.: Magnolia can be not only beatiful, but also helpful. Psychiatrie 6: 247-251, 2002.

Pharmacopoeia of the PRC (English edition). People's Medical Publishing House, Beijing 1988.

Shih H.C., Wei Y.H., Lee C.H.: Magnolol alters the course of endotoxin tolerance and provides early protection against endotoxin challenge following sublethal hemorrhage in rats. Shock 22:358-363, 2004.

Squires R.F., Ai J., Witt M.R. et al.: Honokiol and magnolol increase the number of $\left[{ }^{3} \mathrm{H}\right]$ muscimol binding sites three-fold in rat forebrain membranes in vitro using a filtration assay, by allosterically increasing the affinities of low-affinity sites. Neurochem. Res. 24: 1593-1602, 1999.

Tachikawa E, Takahashi M, Kashimoto T.: Effects of extract and ingredients isolated from Magnolia obovata Thunberg on catecholamine secretion from bovine adrenal chromaffin cells. Biochem. Pharmacol. 60:433-440, 2000.

Tsai S.K., Huang S.S., Hong C.Y.: Myocardial protective effect of honokiol: an active component in Magnolia officinalis. Planta Med. 62:503-506, 1996.

Tsai S.K., Huang C.H., Huang S.S. et al.: Antiarrhythmic effect of magnolol and honokiol during acute phase of coronary occlusion in anesthetized rats: influence of LNAME and aspirin. Pharmacology 59:227233, 1999.

Wang J.P., Ho T.F., Chang L.C., Chen C.C.: Antiinflammatory effect of magnolol, isolated from Magnolia officinalis, on A23187-induced pleurisy in mice. J. Pharm. Pharmacol. 47: 857-860, 1995.

Wang J.P., Hsu M.F., Raung S.L. et al.: Antiinflammatory and analgesic effects of magnolol. Naunyn Schmiedebergs Arch. Pharmacol. 346:707-712, 1992.

Watanabe K., Watanabe H., Goto Y. et al.: Pharmacological properties of magnolol and honokiol extracted from Magnolia officinalis central depressant effects. Planta Med. 49: 103-108, 1983.

Yang M.H., Blunden G., Patel A.V. et al.: Coumarins and sesquiterpene lactones from Magnolia grandiflora leaves. Planta Med. 60:390-390, 1994.

Yang S.E., Hsieh M.T., Tsai T.H., Hsu S.L.: Effector mechanism of magnolol-induced apoptosis in human lung squamous carcinoma 
Patočka et al.: Expectations of biologically active Magnolia compounds

CH27 cells. Br. J. Pharmacol. 138:193-201, 2003.

Zhong W.B., Wang C.Y., Ho K.J. et al.: Magnolol induces apoptosis in human leukemia cells via cytochrome $\mathrm{C}$ release and caspase activation. Anticancer Drugs 14:211-217, 2003. 